\title{
The Influence of Metal Ion Binding on the IR Spectra of Nitrogen-Containing PAHs
}

\author{
Juehan Gao ${ }^{1}$, Jordy Bouwman ${ }^{1}$, Giel Berden ${ }^{1}$ and Jos Oomens ${ }^{1,2 *}$ \\ ${ }^{1}$ Radboud University, Institute for Molecules and Materials, FELIX Laboratory, \\ Toernooiveld 7c, 6525ED Nijmegen, The Netherlands \\ ${ }^{2}$ Van 't Hoff Institute for Molecular Sciences, University of Amsterdam, Science Park \\ 904, 1098XH Amsterdam, The Netherlands
}

\section{SUPPORTING INFORMATION}
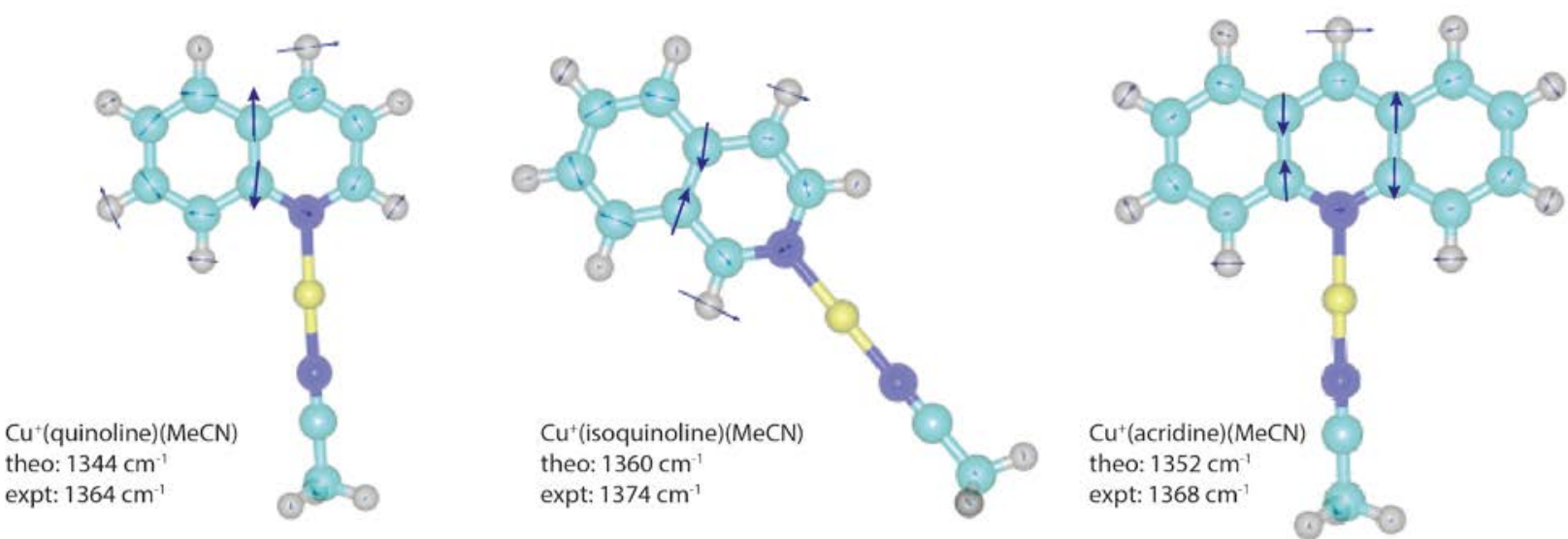

Figure S1: Illustrations of the normal modes giving severe deviation between experimental and theoretical frequencies. In all cases, the normal mode strongly involves stretching of the bond(s) connecting the bridgehead C-atoms. In the $\mathrm{Cu}^{+}(\mathrm{PANH})_{2}$ complexes (not shown), similar modes show similar deviations. 
Table S1. Comparison of observed and computed IR absorption band positions and intensities of neutral quinoline. [a] In $\mathrm{cm}^{-1}$ [b] In $\mathrm{km} / \mathrm{mol}$ [c] Only bands with intensities $>5 \mathrm{~km} / \mathrm{mol}$ are listed.

\begin{tabular}{llll}
\hline Expt freq $^{[a]}$ & Theory freq $^{[a]}$ & int $^{[\mathrm{b}, \mathrm{c}]}$ & description \\
\hline 606 & 605 & 6 & ring ip deform \\
730 & 727 & 10 & CH oop bend \\
786 & 786 & 33 & CH oop bend/ring oop deform \\
802 & 799 & 61 & CH oop bend/ring oop deform \\
1116 & 1104 & 9 & CH ip bend/CCN\& CC stretch/ring deform \\
1320 & 1309 & 8 & CNC \& CC stretch/ CH ip bend/ring deform \\
1370 & 1419 & 5 & CH ip bend/CNC\& CC stretch/ring deform \\
1437 & 1485 & 26 & CH ip bend/CC \& CNC stretch/ring deform \\
1502 & 1545 & 13 & CC\& CNC stretch/ CH ip bend/ring deform \\
& 1580 & 13 & CC stretch/ CH ip bend/CNC stretch/ring deform \\
1594 & 1600 & 7 & CC stretch/ CH ip bend/CNC stretch/ring deform \\
\hline
\end{tabular}

Table S2. Comparison of observed and computed IR absorption band positions and intensities of $\mathrm{Cu}^{+}$(quinoline) ${ }_{1}$. [a] $\mathrm{In}_{\mathrm{cm}}^{-1}$ [b] In $\mathrm{km} / \mathrm{mol}$ [c] Only bands with intensities $>5 \mathrm{~km} / \mathrm{mol}$ are listed.

\begin{tabular}{llll}
\hline $\begin{array}{l}\text { Expt } \\
\text { freq }^{[a]}\end{array}$ & $\begin{array}{l}\text { Theory } \\
\text { freq }^{\text {[a] }}\end{array}$ & int $^{[\mathrm{b}, \mathrm{c}]}$ & \begin{tabular}{l} 
description \\
\hline
\end{tabular} \\
726 & 8 & CH oop bend \\
786 & 766 & 6 & CNC-Cu stretch/ CC stretch/CH ip bend/ ring ip deform \\
& 775 & 38 & CH oop bend/ring oop deform \\
& 797 & 50 & CH oop bend/oop ring deform \\
1138 & 1122 & 8 & CH ip bend/CNC-Cu stretch/ CC stretch/ring ip deform \\
1282 & 1137 & 6 & CH ip bend \\
& 1249 & 13 & CC stretch/CH ip bend/ CNC stretch/ ring ip deform \\
1354 & 1340 & 36 & CNC \& CC stretch/CH ip bend/ ring ip deform \\
& 1448 & 8 & CC stretch/CH ip bend/CNC stretch/ ring ip deform \\
1490 & 1493 & 57 & CC stretch/CH ip bend/CC\&CNC-Cu stretch/ring ip deform \\
& 1553 & 19 & CNC-Cu stretch/ CC stretch/CH ip bend/ ring ip deform \\
& 1567 & 7 & CNC stretch/ CC stretch/CH ip bend/ ring ip deform \\
& 1597 & 12 & CC stretch//CH ip bend/ CNC stretch/ ring ip deform \\
\hline
\end{tabular}


Table S3. Comparison of observed and computed IR absorption band positions and intensities of $\mathrm{Cu}^{+}$(quinoline) ${ }_{2}$. [a] $\mathrm{In}_{\mathrm{cm}}^{-1}$ [b] In $\mathrm{km} / \mathrm{mol}$ [c] Only bands with intensities $>5 \mathrm{~km} / \mathrm{mol}$ are listed.

\begin{tabular}{llll}
\hline $\begin{array}{l}\text { Expt } \\
\text { freq }^{[a]}\end{array}$ & $\begin{array}{l}\text { Theory } \\
\text { freq }^{\text {[a] }}\end{array}$ & int $^{\text {bb,c] }}$ & description \\
\hline 731 & 731 & 6 & CH oop bend/ring oop deform \\
776 & 733 & 6 & CH oop bend/ring oop deform \\
& 769 & 7 & CC \& CNC-Cu stretch/CH ip bend/ ring ip deform \\
& 780 & 36 & CH oop bend/ring oop deform \\
800 & 780 & 38 & CH oop bend/ring oop deform \\
& 802 & 44 & CH oop bend \\
1142 & 802 & 46 & CH oop bend \\
& 1121 & 12 & CH ip bend/CNC-Cu\&CCstretch \\
1294 & 1135 & 6 & CH ip bend \\
& 1222 & 6 & CH ip bend/ CNC-Cu\&CCstretch/ring ip deform \\
1365 & 1296 & 48 & CNC stretch/CC stretch/ CH ip bend/ring ip deform \\
& 1343 & 31 & CC stretch/CH ip bend/CNC stretch/ring ip deform \\
1494 & 1343 & 22 & CC stretch/CH ip bend/CNC stretch/ring ip deform \\
& 1384 & 7 & CC stretch/ CH ip bend/CNC-Cu stretch/ring ip deform \\
& 1450 & 13 & CC stretch/ CH ip bend/CNC-Cu stretch/ring ip deform \\
1574 & 1494 & 74 & CC \& CNC stretch/CH ip bend/ring ip deform \\
& 1495 & 36 & CC \& CNC stretch/CH ip bend/ring ip deform \\
& 1560 & 63 & CC stretch/CNC-Cu stretch/CH ip bend/ring ip deform \\
& 1571 & 10 & CC \&CNC stretch/CH ip bend/rings ip deform \\
& 1600 & 10 & CC stretch/CH ip bend/CNC stretch/ring ip deform \\
& 1600 & 8 & CC stretch/CH ip bend/CNC stretch /ring ip deform \\
\hline
\end{tabular}

Table S4. Comparison of observed and computed IR absorption band positions and intensities of neutral isoquinoline. [a] In $\mathrm{cm}^{-1}$ [b] In $\mathrm{km} / \mathrm{mol}$ [c] Only bands with intensities $>5 \mathrm{~km} / \mathrm{mol}$ are listed.

\begin{tabular}{llll}
\hline Expt freq $^{[\mathrm{a}}$ & Theory freq $^{[\mathrm{a}]}$ & int $^{[\mathrm{b}, \mathrm{c}]}$ & description \\
\hline \multirow{3}{*}{734} & 634 & 5 & ring ip deform \\
822 & 733 & 30 & CH oop bend \\
& 823 & 46 & CH oop bend \\
& 859 & 8 & CH oop bend \\
1014 & 926 & 9 & ring ip deform \\
1266 & 1199 & 5 & CC\&CN stretch/ CH ip bend/ring deform \\
1378 & 1248 & 15 & CC stretch/ CH ip bend/ring deform \\
1498 & 1365 & 7 & CH ip bend/CC stretch /ring deform \\
& 1483 & 10 & CN\& CC stretch/ CH ip bend/ring deform \\
1582 & 1549 & 7 & CN\& CC stretch/ CH ip bend/ring deform \\
1626 & 1568 & 20 & CN\& CC stretch/ CH ip bend/ring deform \\
& 1606 & 21 & CC stretch/ CH ip bend/CN stretch/ring deform \\
\hline
\end{tabular}


Table S5. Comparison of observed and computed IR absorption band positions and intensities of $\mathrm{Cu}^{+}$(isoquinoline) ${ }_{1}$. [a] In $\mathrm{cm}^{-1}$ [b] In $\mathrm{km} / \mathrm{mol}$ [c] Only bands with intensities $>5 \mathrm{~km} / \mathrm{mol}$ are listed.

\begin{tabular}{llll}
\hline $\begin{array}{l}\text { Expt } \\
\text { freq }^{[a]}\end{array}$ & $\begin{array}{l}\text { Theory } \\
\text { freq }^{[a]}\end{array}$ & int $^{[\mathrm{b}, \mathrm{c}]}$ & \begin{tabular}{l} 
description \\
\hline 738
\end{tabular} \\
843 & 25 & CH oop bend/ring oop deform \\
815 & 818 & 49 & CH oop bend \\
& 868 & 11 & CH oop bend/ring oop deform \\
1032 & 1032 & 22 & CNC-Cu stretch/ CC stretch/CH ip bend/ ring ip deform \\
& 1170 & 9 & CH ip bend/ CC\&CNC stretch/ring ip deform \\
1282 & 1262 & 12 & CC stretch/CH ip bend/CNC stretch/ring ip deform \\
& 1362 & 49 & CC\&CNC stretch/CH ip bend/ ring ip deform \\
1374 & 1378 & 19 & CH ip bend/ CNC\&CC stretch/ ring ip deform \\
& 1430 & 6 & CH ip bend/ CNC\&CC stretch/ ring ip deform \\
& 1448 & 14 & CH ip bend/ CNC-Cu \&CC stretch/ ring ip deform \\
1490 & 1484 & 9 & CC stretch/CH ip bend/CNC stretch/ring ip deform \\
& 1580 & 25 & CNC-Cu stretch/CC stretch/CH ip bend/ ring ip deform \\
1597 & 1599 & 49 & CC stretch/CH ip bend/ CNC-Cu stretch/ ring ip deform \\
\hline
\end{tabular}


Table S6. Comparison of observed and computed IR absorption band positions and intensities of $\mathrm{Cu}^{+}$(isoquinoline) ${ }_{2}$. [a] $\mathrm{In} \mathrm{cm}^{-1}$ [b] In $\mathrm{km} / \mathrm{mol}$ [c] Only bands with intensities $>5 \mathrm{~km} / \mathrm{mol}$ are listed.

\begin{tabular}{llll}
\hline $\begin{array}{l}\text { Expt } \\
\text { freq }^{[a]}\end{array}$ & $\begin{array}{l}\text { Theory } \\
\text { freq }^{\text {[a] }}\end{array}$ & int $^{\text {[b,c] }}$ & description \\
\hline & 636 & 7 & Ring oop deform \\
& 636 & 6 & Ring oop deform \\
742 & 742 & 30 & CH oop bend/ ring oop deform \\
818 & 742 & 26 & CH oop bend/ ring oop deform \\
& 823 & 45 & CH oop bend \\
& 823 & 40 & CH oop bend \\
1035 & 866 & 11 & CH oop bend/ ring oop deform \\
& 1035 & 10 & CH oop bend/ ring oop deform \\
& 1169 & 22 & CNC-Cu stretch/ CC stretch/CH ip bend/ring ip \\
1269 & 1202 & 17 & CH ip bend/ CNC\&CC stretch/ring ip deform \\
& 1259 & 23 & CH ip bend/ CNC\&CC stretch/ring ip deform \\
& 1259 & 7 & CC stretch/ CH ip bend/ rings ip deform \\
& 1311 & 6 & CC stretch/ CH ip bend/ rings ip deform \\
& 1360 & 78 & CNC\&CC stretch/ CH ip bend/ ring ip deform \\
1372 & 1360 & 9 & CC\&CNC stretch/ CH ip bend/ ring ip deform \\
& 1375 & 18 & CH ip bend/ CC stretch/ ring ip deform \\
& 1376 & 14 & CH ip bend/ CC stretch/ ring ip deform \\
& 1427 & 5 & CC \& CNC stretch/CH ip bend/ring ip deform \\
& 1449 & 20 & CC\&CNC-Cu stretch/ CH ip bend/ ring ip deform \\
& 1485 & 8 & CC\&CNC stretch/ CH ip bend/ ring ip deform \\
& 1485 & 8 & CC\&CNC stretch/ CH ip bend/ ring ip deform \\
& 1582 & 12 & CC\&CNC-Cu stretch/ CH ip bend/ ring ip deform \\
& 1582 & 29 & CC\&CNC-Cu stretch/ CH ip bend/ ring ip deform \\
& 1605 & 110 & CC stretch /CH ip bend/CNC-Cu stretch ring ip \\
& & & deform \\
\hline
\end{tabular}


Table S7. Comparison of observed and computed IR absorption band positions and intensities of neutral acridine. [a] $\mathrm{In}_{\mathrm{cm}}^{-1}$ [b] In $\mathrm{km} / \mathrm{mol}$ [c] Only bands with intensities $>5 \mathrm{~km} / \mathrm{mol}$ are listed.

\begin{tabular}{llll}
\hline Expt freq $^{[a\rfloor}$ & Theory freq $^{[\mathrm{a}\rfloor}$ & int $^{[\mathrm{b,c}]}$ & description \\
\hline 734 & 729 & 103 & CH oop bend \\
784 & 852 & 15 & CH oop bend \\
& 853 & 6 & CH oop bend/ring oop deform \\
902 & 910 & 15 & CH oop bend/ring oop deform \\
& 959 & 6 & CH oop bend/ring oop deform \\
& 987 & 7 & CH ip bend/CC stretch/ring ip deform \\
1138 & 1127 & 10 & CH ip bend \\
1394 & 1370 & 6 & CC\& CNC stretch/CH ip bend/ring ip deform \\
1466 & 1502 & 46 & CC\& CNC stretch/CH ip bend/ring ip deform \\
1514 & 1532 & 9 & CC\& CNC stretch/CH ip bend/ring ip deform \\
1560 & 1562 & 9 & CC\& CNC stretch/CH ip bend/ring ip deform \\
1620 & 1595 & 19 & CC\& CNC stretch/CH ip bend/ring ip deform \\
\hline
\end{tabular}

Table S8. Comparison of observed and computed IR absorption band positions and intensities of $\mathrm{Cu}^{+}$(acridine) ${ }_{1}$. [a] In $\mathrm{cm}^{-1}$ [b] In km/mol [c] Only bands with intensities $>5 \mathrm{~km} / \mathrm{mol}$ are listed.

\begin{tabular}{llll}
\hline $\begin{array}{l}\text { Expt } \\
\text { freq }^{[a]}\end{array}$ & $\begin{array}{l}\text { Theory } \\
\text { freq }^{[a]}\end{array}$ & int $^{[\mathrm{b}, \mathrm{c}]}$ & description \\
\hline 725 & 729 & 83 & CH oop bend \\
768 & 776 & 32 & CH oop bend/ring oop deform \\
& 819 & 8 & CH oop bend/CC stretch/ring oop deform \\
& 934 & 12 & CH oop bend/ring oop deform \\
& 968 & 6 & CH oop bend \\
1145 & 1134 & 7 & CH ip bend/CNC stretch \\
& 1142 & 18 & CH ip bend/CNC-Cu stretch \\
& 1158 & 7 & CH ip bend \\
& 1166 & 6 & CH ip bend/ring ip deform \\
& 1245 & 6 & CNC-Cu stretch/CC stretch/CH ip bend/ring ip deform \\
1362 & 1262 & 25 & CH ip bend/ring ip deform/N-Cu stretch \\
1439 & 1348 & 3 & CNC stretch/CH ip bend/ring ip deform \\
& 1427 & 8 & CC stretch/CH ip bend/ ring ip deform/CNC stretch \\
1508 & 1443 & 19 & CH ip bend/ ring ip deform/CC\&CNC stretch \\
& 1508 & 92 & CC\& CNC stretch/CH ip bend/ ring ip deform stretch/CH ip bend/ ring ip deform \\
1559 & 1555 & 13 & CC stretch/CNC-Cu stretch/CH ip bend/ring ip deform \\
1592 & 1591 & 77 & CC stretch/CNC-Cu stretch/CH ip bend/ring ip deform \\
\hline
\end{tabular}


Table S9. Comparison of observed and computed IR absorption band positions and intensities of $\mathrm{Cu}^{+}$(acridine) ${ }_{2}$. [a] In $\mathrm{cm}^{-1}$ [b] In $\mathrm{km} / \mathrm{mol}$ [c] Only bands with intensities $>5 \mathrm{~km} / \mathrm{mol}$ are listed.

\begin{tabular}{|c|c|c|c|}
\hline Expt freq $^{[a]}$ & Theory freq $^{\text {[a] }}$ & int $^{[b, c]}$ & description \\
\hline & 661 & 7 & ring ip deform/ N-Cu-N stretch \\
\hline \multirow[t]{2}{*}{732} & 735 & 74 & $\mathrm{CH}$ oop bend \\
\hline & 735 & 74 & CH oop bend \\
\hline \multirow[t]{3}{*}{777} & 769 & 7 & $\mathrm{CH}$ ip bend/ ring ip deform/ N-Cu-N stretch \\
\hline & 783 & 20 & $\mathrm{CH}$ oop bend/ ring oop deform \\
\hline & 783 & 20 & $\mathrm{CH}$ oop bend/ ring oop deform \\
\hline \multirow[t]{4}{*}{918} & 931 & 14 & $\mathrm{CH}$ oop bend/ ring oop deform \\
\hline & 931 & 14 & $\mathrm{CH}$ oop bend/ ring oop deform \\
\hline & 1131 & 6 & $\mathrm{CH}$ ip bend/ ring ip deform/ C-N-C stretch \\
\hline & 1131 & 6 & $\mathrm{CH}$ ip bend/ ring ip deform/ C-N-C stretch \\
\hline \multirow[t]{3}{*}{1152} & 1142 & 20 & $\mathrm{CH}$ ip bend / N-Cu-N stretch \\
\hline & 1246 & 22 & $\mathrm{CH}$ ip bend/ ring ip deform/ CNC-Cu stretch \\
\hline & 1266 & 15 & $\mathrm{CH}$ ip bend/ ring ip deform \\
\hline \multirow[t]{4}{*}{1374} & 1352 & 25 & $\mathrm{CH}$ ip bend/ ring ip deform/CC\&CNC stretch \\
\hline & 1352 & 25 & $\mathrm{CH}$ ip bend/ ring ip deform/CC\&CNC stretch \\
\hline & 1429 & 7 & $\mathrm{CH}$ ip bend/ ring ip deform/ CC stretch \\
\hline & 1429 & 7 & $\mathrm{CH}$ ip bend/ ring ip deform/ CC stretch \\
\hline \multirow[t]{2}{*}{1449} & 1445 & 52 & $\mathrm{CH}$ ip bend/ ring ip deform/ CC stretch \\
\hline & 1469 & 14 & $\mathrm{CH}$ ip bend/ ring ip deform/ CC stretch \\
\hline \multirow[t]{3}{*}{1509} & 1506 & 76 & CC \& CNC stretch/CH ip bend/ring ip deform \\
\hline & 1506 & 76 & CC \& CNC stretch/CH ip bend/ring ip deform \\
\hline & 1541 & 62 & CC \& CNC stretch/CH ip bend/ring ip deform \\
\hline \multirow[t]{2}{*}{1560} & 1559 & 17 & CC \& CNC stretch/CH ip bend/ring ip deform \\
\hline & 1559 & 17 & CC \& CNC stretch/CH ip bend/ring ip deform \\
\hline \multirow[t]{2}{*}{1602} & 1596 & 58 & CC \& CNC stretch/CH ip bend/ring ip deform \\
\hline & 1596 & 58 & CC \& CNC stretch/CH ip bend/ring ip deform \\
\hline
\end{tabular}

Table S10. Comparison of observed IR absorption bands of neutral quinoline, $\mathrm{Cu}^{+}$(quinoline) ${ }_{1}$, $\mathrm{Cu}^{+}$(quinoline) $)_{2}$, and $\mathrm{Cu}^{+}$(quinoline)(MeCN).

\begin{tabular}{|c|c|c|c|}
\hline $\begin{array}{l}\text { Exp. freq. }\left(\mathrm{cm}^{-1}\right) /(\mu \mathrm{m}) \\
\text { Neutral quinoline }^{\mathrm{a}}\end{array}$ & $\begin{array}{l}\text { Exp. freq. }\left(\mathrm{cm}^{-1}\right) /(\mu \mathrm{m}) \\
\left.\mathrm{Cu}^{+} \text {(quinoline }\right)_{1}\end{array}$ & $\begin{array}{l}\text { Exp. freq. }\left(\mathrm{cm}^{-1}\right) /(\mu \mathrm{m}) \\
\left.\mathrm{Cu}^{+} \text {(quinoline }\right)_{2}\end{array}$ & $\begin{array}{l}\text { Exp. freq. }\left(\mathrm{cm}^{-1}\right) /(\mu \mathrm{m}) \\
\mathrm{Cu}^{+} \text {(quinoline)MeCN }\end{array}$ \\
\hline $786 / 12.72$ & \multirow{2}{*}{$786 / 12.72$} & $776 / 12.89$ & $776 / 12.89$ \\
\hline $802 / 12.47$ & & $800 / 12.50$ & $800 / 12.50$ \\
\hline $1322 / 7.56$ & $1282 / 7.80$ & $1294 / 7.73$ & $1293 / 7.73$ \\
\hline $1370 / 7.30$ & $1354 / 7.39$ & $1365 / 7.33$ & $1364 / 7.33$ \\
\hline $1502 / 6.66$ & $1490 / 6.71$ & $1494 / 6.69$ & $1496 / 6.68$ \\
\hline $1594 / 6.27$ & - & $1574 / 6.35$ & $1579 / 6.33$ \\
\hline
\end{tabular}

${ }^{\mathrm{a}}$ Frequencies are taken from NIST. 
Table S11. Comparison of observed IR absorption bands of neutral isoquinoline, $\mathrm{Cu}^{+}$(isoquinoline) ${ }_{1}, \mathrm{Cu}^{+}$(isoquinoline) ${ }_{2}$, and $\mathrm{Cu}^{+}$(isoquinoline) $(\mathrm{MeCN})$.

\begin{tabular}{|l|l|l|l|}
\hline $\begin{array}{l}\text { Exp.freq. }\left(\mathrm{cm}^{-1}\right) /(\mu \mathrm{m}) \\
\text { Neutral isoquinoline }\end{array}$ & $\begin{array}{l}\text { Exp.freq. }\left(\mathrm{cm}^{-1}\right) /(\mu \mathrm{m}) \\
\mathrm{Cu}^{+}(\text {isoquinoline })_{1}\end{array}$ & $\begin{array}{l}\text { Exp.freq. }\left(\mathrm{cm}^{-1}\right) /(\mu \mathrm{m}) \\
\mathrm{Cu}^{+}(\text {isoquinoline })_{2}\end{array}$ & $\begin{array}{l}\text { Exp.freq. }\left(\mathrm{cm}^{-1}\right) /(\mu \mathrm{m}) \\
\mathrm{Cu}^{+}(\text {isoquinoline}) \mathrm{MeCN}\end{array}$ \\
\hline $734 / 13.62$ & $738 / 13.55$ & $742 / 13.48$ & $741 / 13.50$ \\
\hline $822 / 12.17$ & $815 / 12.27$ & $818 / 12.22$ & $821 / 12.18$ \\
\hline $1014 / 9.86$ & $1032 / 9.69$ & $1035 / 9.66$ & $1041 / 9.61$ \\
\hline $1266 / 7.90$ & - & $1269 / 7.88$ & $1273 / 7.86$ \\
\hline $1378 / 7.26$ & $1374 / 7.28$ & $1372 / 7.29$ & $1374 / 7.28$ \\
\hline $1498 / 6.68$ & $1490 / 6.71$ & $1494 / 6.69$ & $1496 / 6.68$ \\
\hline $1582 / 6.32$ & $1597 / 6.26$ & $1596 / 6.27$ & $1615 / 6.19$ \\
\hline $1626 / 6.15$ & & & \\
\hline
\end{tabular}

${ }^{\mathrm{a}}$ Frequencies are taken from NIST.

Table S12. Comparison of observed IR absorption bands of neutral acridine, $\mathrm{Cu}^{+}(\text {acridine })_{1}$, $\mathrm{Cu}^{+}$(acridine) $)_{2}$, and $\mathrm{Cu}^{+}$(acridine) $(\mathrm{MeCN})$.

\begin{tabular}{|c|c|c|c|}
\hline $\begin{array}{l}\text { Exp.freq. }\left(\mathrm{cm}^{-1}\right) /(\mu \mathrm{m}) \\
\text { Neutral acridine }^{\mathrm{a}}\end{array}$ & $\begin{array}{l}\text { Exp.freq. }\left(\mathrm{cm}^{-1}\right) /(\mu \mathrm{m}) \\
\left.\mathrm{Cu}^{+} \text {(acridine }\right)_{1}\end{array}$ & $\begin{array}{l}\text { Exp.freq. }\left(\mathrm{cm}^{-1}\right) /(\mu \mathrm{m}) \\
\mathrm{Cu}^{+} \text {(acridine) } \\
\end{array}$ & $\begin{array}{l}\text { Exp.freq. }\left(\mathrm{cm}^{-1}\right) /(\mu \mathrm{m}) \\
\mathrm{Cu}^{+} \text {(acridine)MeCN }\end{array}$ \\
\hline 734/13.62 & 725/13.79 & $732 / 13.66$ & $732 / 13.66$ \\
\hline $784 / 12.76$ & $768 / 13.02$ & $777 / 12.87$ & $777 / 12.87$ \\
\hline $902 / 11.09$ & - & $918 / 10.89$ & $921 / 10.86$ \\
\hline $1138 / 8.79$ & $1145 / 8.73$ & $1152 / 8.68$ & $1151 / 8.69$ \\
\hline $1394 / 7.17$ & $1362 / 7.34$ & $1374 / 7.28$ & 1368/7.31 \\
\hline 1466/6.82 & $1439 / 6.95$ & 1449/6.90 & $1453 / 6.88$ \\
\hline $1514 / 6.61$ & $1508 / 6.63$ & $1509 / 6.63$ & $1510 / 6.22$ \\
\hline $1560 / 6.41$ & $1559 / 6.41$ & $1560 / 6.41$ & $1558 / 6.42$ \\
\hline $1620 / 6.17$ & $1592 / 6.28$ & $1602 / 6.24$ & $1604 / 6.23$ \\
\hline
\end{tabular}

${ }^{\mathrm{a}}$ Frequencies are taken from NIST. 